\title{
Dust around red supergiants in the Magellanic Clouds
}

\author{
Geoffrey C. Clayton ${ }^{1}$, W. Freeman ${ }^{1}$, S. Bright ${ }^{1}$, P. Massey ${ }^{2}$,

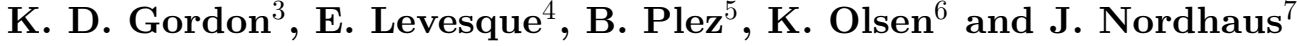 \\ ${ }^{1}$ Department of Physics \& Astronomy, Louisiana State University, Baton Rouge, LA 70803, \\ USA \\ email: gclayton@fenway.phys.lsu.edu \\ ${ }^{2}$ Lowell Observatory, 1400 W Mars Hill Rd., Flagstaff, AZ 86001, USA \\ email: phil.massey@lowel.edu \\ ${ }^{3}$ Space Telescope Science Institute, 3700 San Martin Drive Baltimore, MD 21218 \\ email: kgordon@stsci.edu \\ ${ }^{4}$ Institute for Astronomy, University of Hawaii, 2680 Woodlawn Drive, Honolulu, HI 96822, \\ USA \\ email: emsque@ifa.hawaii.edu \\ ${ }^{5}$ GRAAL, Universite de Montpellier II, CNRS, 34095 Montpellier, France \\ email: bertrand.plez@graal.univ-montp2.fr \\ ${ }^{6}$ Cerro Tololo Inter-American Observatory, NOAO, Casilla 603, La Serena, Chile \\ email: kolsen@noao.edu \\ ${ }^{7}$ Department of Astrophysical Sciences, Princeton University, Princeton, NJ 08544, USA \\ email: nordhaus@astro.princeton.edu
}

\begin{abstract}
It is both surprising and exciting to find that young galaxies at high redshift contain large dust masses. For galaxies at $z>5$, after only $1 \mathrm{Gyr}$, there has not been time for low-mass stars to have evolved to the AGB phase and produce dust. In such galaxies, Type II SNe and red supergiants (RSGs) may even dominate the dust production rate. It has long been known that RSG atmospheres produce dust, but little is known about it. We are pursuing three parallel studies to better understand RSG dust. First, we are using optical spectra and JHK photometry to characterize the optical and near-IR extinction curves of the RSGs. Second, we are using the optical spectra combined with 2MASS, IRAC and MIPS photometry to estimate the dust mass loss rates from Local Group RSGs. In addition, we will use our Monte Carlo radiative transfer models to analyze the emission from dust in the circumstellar shells. Third, the final piece of the puzzle is being provided by obtaining new IRS spectra of LMC and SMC RSGs. We plan to use the IRS to make a systematic study of the dust properties in RSG shells in the LMC and SMC so that we can probe how they may vary with a large range of galactic metallicities. The derived stellar SEDs and extinction curves will be combined with Spitzer IRAC and MIPS photometry and IRS spectra for use as inputs to our Monte Carlo codes which will be used to study the composition, size distributions and clumpiness of the dust.
\end{abstract}

Keywords. circumstellar matter, supergiants, dust, extinction, Magellanic Clouds

\section{Introduction}

Red Supergiants (RSGs) are the evolved, He-burning descendants of moderately massive $\left(\leqslant 40 \mathrm{M}_{\odot}\right) \mathrm{O}$ and B stars. In reviews of the origin of cosmic dust, the role of RSGs is often ignored, with the primary sources of dust given as SNe and low-mass AGB stars. However, for primordial galaxies, RSG dust will play a more important role due the absence of old low-mass AGB stars. So, in extreme environments (such as primordial 



Figure 1. Two of our target RSGs, LMC 119219 (left) and LMC 116895 (right). The plotted spectrum to the blue of $1 \mu \mathrm{m}$ is the visible spectrum. The triangles are the 2MASS JHK, and the diamonds are the IRAC 3.6, 4.5, $5.8 \& 8.0 \mu \mathrm{m}$, and MIPS $24 \mu \mathrm{m}$ photometry. The solid line in the IR is the Spitzer/IRS spectrum.

galaxies, lacking in AGBs), we expect that RSGs may be a primary source of dust along with the Type II SNe (Massey et al. 2005).

It has long been known that RSG atmospheres are "smokey" and produce dust at distances of 5-10 stellar radii from the star (Danchi et al. 1994), but it has only just been realized that a significant fraction of RSGs in Galactic OB associations and clusters show several magnitudes of excess visual extinction compared to OB stars in the same regions. Massey et al. (2005) demonstrate that this is in fact just what we should expect, given the amount of dust produced by RSGs (Josselin et al. 2000). Furthermore, the stars with the highest amount of extra visual extinction also show significant near-UV (NUV) excesses compared to MARCs stellar atmosphere modes (Plez et al. 1992) reddened by the standard reddening law. The discrepancy in the NUV is striking. The size of the NUV discrepancy is well correlated with the amount of extra extinction, the dust production rate $\dot{M}_{\mathrm{d}}$ as measured from the $12-\mu \mathrm{m}$ excess, and the bolometric luminosity of the star computed from the K-band (Massey et al. 2005).

\section{Infrared emission from dust around red supergiants}

We have been amassing a unique dataset which can be used to investigate the amount and nature of dust produced by RSGs in very different environments. To this end, optical spectra are being obtained for RSGs in various galaxies in the Local Group. Moderateresolution, high $\mathrm{S} / \mathrm{N}$, spectrophotometry has been obtained of $36 \mathrm{RSG}$ in the LMC and 37 RSGs in the SMC (Levesque et al. 2006). These spectra were taken using the CTIO Blanco $4 \mathrm{~m}$ telescope and cover the range 3500-9000 $\AA$ (Figure 1). Similar highquality data were recently obtained for a sample of M31 RSGs using the MMT $6.5 \mathrm{~m}$. It is planned to expand the sample to RSGs in M33 and NGC 6822 in the near future. The stars in our sample have been definitively identified as RSGs by relying on radial velocities to distinguish foreground dwarfs from RSGs in the LMC, SMC and M 31.

In order to investigate dust around the RSGs, we need to measure their emission in the infrared. High energy photons from the central star warms dust lying in a surrounding 


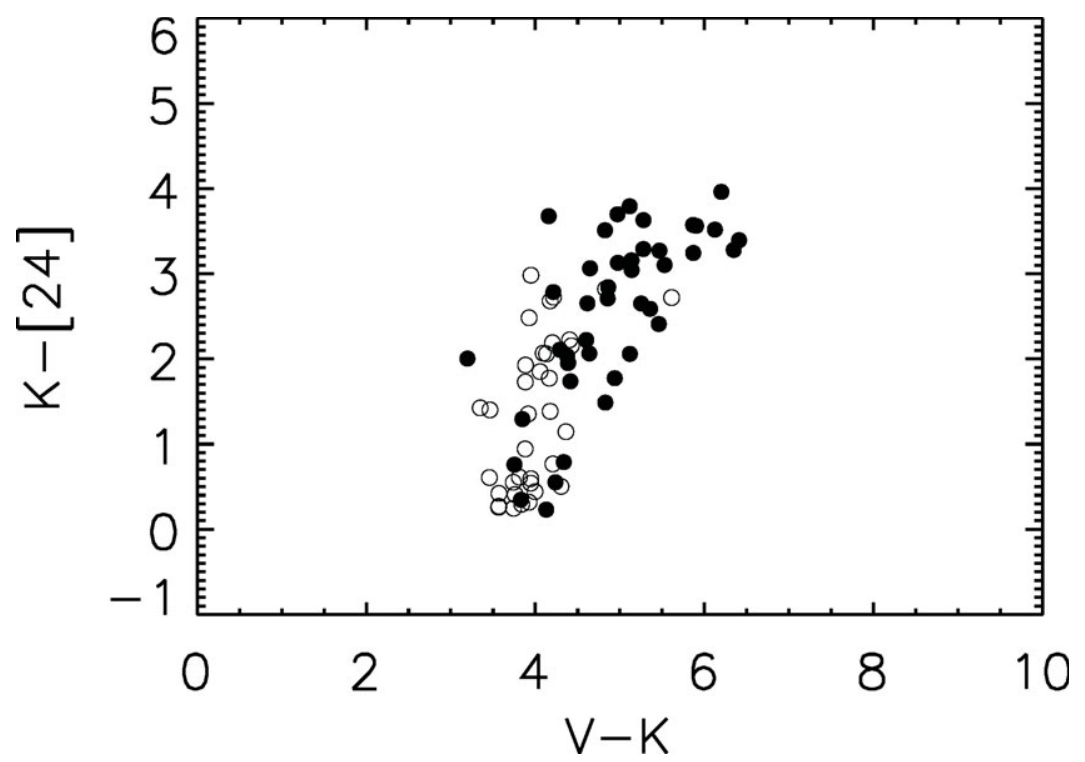

Figure 2. Colour-colour plot of LMC (filled circles) and SMC (open circles) RSGs. The $K-[12]$ color has been used to estimate dust mass-loss rates (Josselin et al. 2000). We plan to calibrate the $K-[24]$ color using the MIPS $24 \mu \mathrm{m}$ photometry.

shell. The warm dust re-emits this energy in the IR. Near-IR photometry in the JHK bands is available from the 2MASS archive, as well as new JHK photometry for the M31 stars we obtained using the KPNO $4 \mathrm{~m}$ telescope. In addition, we have a Spitzer archival program to get IRAC and MIPS photometry of RSGs in Local Group galaxies. We will use the derived stellar SEDs and IR photometry as inputs to the radiative transfer codes described below. By measuring the amount of dust produced by RSGs in different environments (low vs. high metallicity), we can quantify their role in galactic dust-content evolution, which will have an impact on our understanding of massive star evolution and dust production. In particular, we will quantify the circumstellar infrared excess, and the relationship of mass loss to stellar properties, using the $K-[24]$ colour index as a measure of mass loss similar to what has been done previously with the $K-[12]$ colours (Josselin et al. 2000). See Figure 2.

\section{Modeling}

The size, shape, and composition of the dust grains are inputs to the radiative transfer models. We will apply the DIRTY Monte Carlo code (Misselt et al. 2001; Gordon et al. 2001) to model radiative transfer in the circumstellar shells around the RSGs. We expect that many of our sample RSGs in the LMC, SMC and M 31 will have significant detections in the IRAC bandpasses, as well as at $24 \mu \mathrm{m}$ with MIPS. Figure 1 shows the IR excess from the shells around two of the LMC stars in our sample. The stellar SEDs and extinction curves, derived from the optical and near-IR data, will be combined with the Spitzer IR photometry to use as inputs to the radiative transfer codes. We will model the dust shells using amorphous carbon and silicate dust grains as well as Polycyclic aromatic hydrocarbons molecules (PAHs) appropriate to these shells. This modeling will allow us to infer the total circumstellar dust mass around the sample RSGs. The estimated optical 
depth of the dust will also allow us to assess the level of contamination of the extinction curves by scattered light in the NUV.

We will look for differences in the nature of this dust, its mass, composition, size distribution, extinction and re-emission properties between the LMC, SMC and M 31. In particular, we will consider whether the large global metallicity differences between these three galaxies lead to observable differences in the dust being produced by their RSGs.

\section{References}

Danchi, W. C., Bester, M., Degiacomi, C. G., Greenhill, L. J., \& Townes, C. H. 1994, AJ, 107, 1469

Gordon, K. D., Misselt, K. A., Witt, A. N., \& Clayton, G. C. 2001, ApJ, 551, 269

Josselin, E., Blommaert, J. A. D. L., Groenewegen, M. A. T., Omont, A., \& Li, F. L. 2000, $A \& A, 357,225$

Levesque, E. M., Massey, P., Olsen, K. A. G., Plez, B., Meynet, G., \& Maeder, A. 2006, ApJ, 645,1102

Massey, P., Plez, B., Levesque, E. M., Olsen, K. A. G., Clayton, G. C., \& Josselin, E. 2005, ApJ, 634, 1286

Misselt, K. A., Gordon, K. D., Clayton, G. C., \& Wolff, M. J. 2001, ApJ, 551, 277

Plez, B., Brett, J. M., \& Nordlund, Å. 1992, A\&A, 256, 551

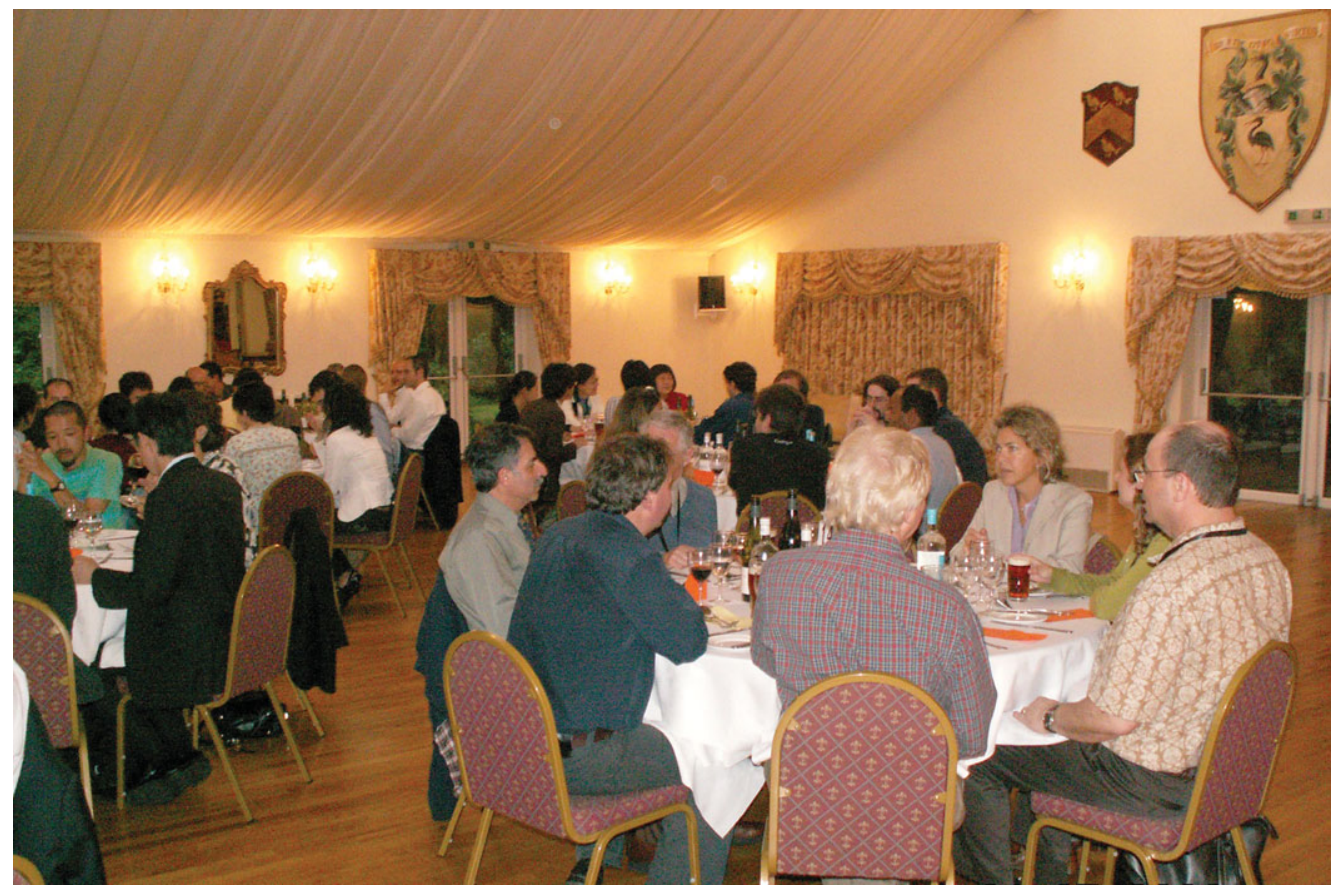

At the conference dinner inside Wrenbury Hall. 